\title{
Heating, Cooling, and Equilibration of an Interacting Many-Fermion System
}

\author{
Angel Ricardo Plastino', Gustavo Luis Ferri², Angelo Plastino ${ }^{3}$ \\ ${ }^{1}$ CeBio-Departamento de Ciencias Básicas, Universidad Nacional del Noroeste de la Prov. de Buenos Aires (UNNOBA), \\ CONICET, Junin, Argentina \\ ${ }^{2}$ Departamento de Física, Facultad de Ciencias Exactas y Naturales, Universidad Nacional de La Pampa, \\ Santa Rosa, Argentina \\ ${ }^{3}$ Universidad Nacional de La Plata (UNLP), IFLP-CCT-CONICET, La Plata, Argentina \\ Email: angeloplastino@gmail.com
}

How to cite this paper: Plastino, A.R., Ferri, G.L. and Plastino, A. (2020) Heating, Cooling, and Equilibration of an Interacting Many-Fermion System. Journal of Modern Physics, 11, 1312-1325.

https://doi.org/10.4236/jmp.2020.119082

Received: August 12, 2020

Accepted: September 11, 2020

Published: September 14, 2020

Copyright $\odot 2020$ by author(s) and Scientific Research Publishing Inc. This work is licensed under the Creative Commons Attribution International License (CC BY 4.0).

http://creativecommons.org/licenses/by/4.0/

\begin{abstract}
We discuss the process of equilibrium's attainment in an interacting manyfermions system linked to a heat reservoir, whose temperature $T$ is subject to a short-time disturbance of total duration $2 \tau$. In this time-interval, its temperature increases up to a maximum value $T_{M}$, cooling off afterward (also gradually) to its original value. The process is described by a typical master equation that leads eventually to equilibration. We discuss how the equilibration process depends upon 1) the system's fermion-number, 2) the fermionfermion interaction's strength $V, 3$ ) the disturbance duration $2 \tau$, and finally 4) the maximum number of equations $N$ of the master equation.
\end{abstract}

\section{Keywords}

Many-Fermion System, Master Equation, Temperature Disturbances, Equilibration Process

\section{Introduction}

\subsection{Preliminaries}

People use master equations (ME) to obtain equations of motion for a reduced density operator or for the probability distribution (PD) of a subsystem of interest $\mathcal{S}$ in interaction with (a usually much larger) heath bath. The key topic is that $\mathcal{S}$, our system at hand, is in an out of equilibrium state. Master equations are standard tools in statistical physics and related areas. See for instance [1] and references therein. The core issue is how to gather relevant information on $\mathcal{S}$ from the concomitant out of equilibrium dynamics. Thus, our goal is to describe 
the time evolution of the PD for the system $\mathcal{S}$. This should be done by recourse to a master equation complying with some basic requirements. First, the concomitant dynamics guarantees positivity and normalization of the time-dependent PD. Second, for a constant heat-bath temperature, the PD satisfies an appropriate $\mathrm{H}$-theorem consistent with the PD's relaxation towards equilibrium. Last, for a constant heat-bath temperature, the associated Gibbs' distribution is a stationary solution of the master equation. The solution to this problem is found by recourse to an appropriate master equation (ME) [1]. A nice instantiation of the ME-procedure is presented by Takada, Conradt, and Richet (TCR) in [2], that we discuss below for didactic purposes.

\subsection{TCR Approach}

Contemplate a two-levels ( 1 and 2) model (system $\mathcal{S}$ ) in contact with a heath bath $\mathrm{B}$ of temperature $T$. If the population $\mathcal{P}_{2}$ of the excited state is calculated, then $\mathcal{P}_{1}$ becomes automatically determined. The inter-level transition rate is the critical parameter ruling the degree of non-equilibrium. We call $p_{1}$ and $p_{2}$ the two occupation probabilities.

Now imagine a heating and cooling operation in which the reservoir's temperature varies as with time in this manner: 1) $T$ first grows, reaches a maximum value $T_{M}$ (arbitrary units (AU)), and then 2) diminishes, returning to its initial value $T_{0}=1(\mathrm{AU})$. TCR interpret this environment as describing a particle movable in an asymmetric double-well potential. Site 1 is the bottom of the first well (of energy is $E_{1}$ ). Analogously, site 2 is the bottom of a second well, of a higher energy $E_{2}$. Thus, $E_{1}$ is a potential energy barrier between states 1 and 2 to be overcome. System $S$ evolves after wards with an energy decrease to $E_{2}$, leading to the second state. The temperature describes an isosceles time-triangle of apex $T_{M}$ and base $2 \tau$. Takada, Conradt, and Richet concoct the concomitant master equation (ME) as

$$
\mathrm{d} p_{1} / \mathrm{d} t=-a_{1} p_{1}+a_{2} p_{2} ; p_{1}+p_{2}=1,
$$

with

$$
a_{i}=\exp \left[-E_{i} / k_{b} T\right], i=1,2,
$$

where $k_{B}$ is Boltzmann's constant, to be set equal to unity here from.

\subsection{Present Objectives}

Inspired by [2], we will tackle a different problem. We focus attention on a quantum many-body system $\mathcal{S}$ of interacting fermions, proposed in [3], whose interaction is governed by an SU2 algebra. $\mathcal{S}$ is first heated and then cooled in the time-triangle fashion described above. We will deal with a master coupled system of $N$ equations (not just two as in [2]). The heating-cooling operation competes with the fermion-fermion interaction. A Gibbs' canonical ensemble treatment of the model advanced in [3] is detailed in [4]. At the same time, for each distinct temperature $T$ we contemplate a fictional coupled system $\mathcal{S}$-heat bath in thermal equilibrium at any extant $T$-value [this is called the quasi-static 
approximation (qsa)]. Of course, at that time $t$ at which the solutions of our master equation coincide with those of the qsa we will have reached equilibrium.

Our main research tools will be suitable information theory quantifiers. With them we will assess "distances" between the "ME probability distribution" (MEPD) and the "quasi-static" PD. The ensuing numerical values will indicate just different the two treatments MEPD and qsa are. This yields a quantitative measure of how far on is from equilibrium. To objectively measure this is our central goal here. Epistemology teaches classification is an essential ingredient of Science [5]. Classification entails numbers, and the distances that we are seeking do supply such numbers in the present context.

\subsection{Paper's Organization}

Section 2 describes our master equation. Suitable details of our exactly solvable quantum many-body system, (the laboratory to test our thermal distances between off equilibrium and equilibrium) are the subject of Section 3. Section 4 discusses features about thermal quantifiers that will serve as "distances". The main present results are displayed exhibited and talked through in Section 5 and, finally, some conclusions are elaborated in Section 6.

\section{Fully Interacting Master Equation}

We advance here our master equation (ME). In it, all energy states $j$ of probability $p_{j}$ interact with each other, so that the ensuing differential equation becomes, for $j=0, \cdots, N-1$,

$$
\frac{\mathrm{d} p_{i}}{\mathrm{~d} t}=\sum_{j=0 ; j \neq i}^{N-1} c_{i j}\left[p_{j} \exp \left(-\beta E_{i}\right)-p_{i} \exp \left(-\beta E_{j}\right)\right],
$$

where the energy spectrum is given by the $\left\{E_{i}=i\right\}$ and $\beta=1 / k_{B} T$. The matrix $C$ of the $c_{i j}$ is symmetric with non-negative elements. $c_{i i}=0$ for all diagonal elements. The initial conditions are $p_{i}(t=0)=\exp \left(-E_{i} / k T\right) / Z$. for all $i$. This ME has nice properties.

- If at $t=t_{0}$ we have $p_{i}\left(t_{0}\right)=0$, then $\frac{\mathrm{d} p_{i}\left(t_{0}\right)}{\mathrm{d} t} \geq 0$.

- The Gibbs distribution $p_{i}^{G}=\exp \left(-\beta E_{i}\right) / Z$, with

$$
\sum_{i=0}^{N-1} \exp \left(-\beta E_{i}\right)=Z
$$

is a stationary solution of our ME.

- Normalization is preserved because

$$
\sum_{i=0}^{N-1} \frac{\mathrm{d} p_{i}}{\mathrm{~d} t}=0
$$

as we prove below.

\subsection{Normalization Proof}

Introduce first an antisymmetric matrix $A$ whose elements $A_{i j}$ have the fol- 
lowing properties:

$$
A_{i j}=\sum_{i, j=1}^{N}\left[p_{j} \exp \left(-\beta E_{i}\right)-p_{i} \exp \left(-\beta E_{j}\right)\right],
$$

so that $A_{i j}=-A_{j i}$. Then,

$$
\frac{\sum_{i=1}^{N} p_{i}}{\mathrm{~d} t}=\sum_{i, j=1}^{N} c_{i j} A_{i j}=\sum_{i, j=1 ; i}^{N} c_{l e j}\left(A_{i j}+A_{j i}\right)=0,
$$

q.e.d.

\subsection{A Simplified Master Equation}

A much simpler ME was recently studied in Ref. [6]. Here we permit interaction only between neighbor levels (with one exception demanded by normalization). This entails some sort of selection rules that allow for only certain kinds of transitions. The simplified ME reads

$$
\frac{\mathrm{d} p_{k}}{\mathrm{~d} t}=p_{k-1} \exp \left(-\beta E_{k}\right)-p_{k} \exp \left(-\beta E_{k-1}\right),
$$

and we continue in this manner till we encounter

$$
\begin{aligned}
\frac{\mathrm{d} p_{0}}{\mathrm{~d} t}= & -\left[p_{n-1} \exp \left(-\beta E_{n}\right)-p_{n} \exp \left(-\beta E_{n-1}\right)\right]-\cdots \\
& -\left[p_{0} \exp \left(-\beta E_{1}\right)-p_{1} \exp \left(-\beta E_{0}\right)\right],
\end{aligned}
$$

where the last relation guarantees normalization. The initial conditions are $p_{i}(0)=\exp \left(-E_{i} / k T\right) / Z$. for all $i . \quad \beta$ depends upon time in the peculiar triangular way described above. We set

$$
\beta(t)=1 / T(t),
$$

together with

$$
E_{j}=j ; \text { all } j \text {. }
$$

If $\beta$ were constant, then the time-dependent probabilities $p_{i}(t)$ would relax to stationary Gibbs-distributions. We will compare below results, in particular equilibrium attainment, for this simpler ME with those yielded by the more complete ME described above.

\section{M-Fermions' Exactly Solvable Model}

We contemplate $M$ fermions distributed amongst $(2 M)$-fold degenerate single-particle (sp) levels, separated by a sp energy gap $\epsilon$ [3] [4]. Two quantum numbers $\mu$ and $p$ are affixed to a generic single particle state. We have the values $\mu=-1$ (lower level) and $\mu=+1$ (upper level). The quantum number $p$, denominated quasi-spin, selects a state out of the $M$-fold degeneracy. The pair $p, \mu$ can also be regarded as a "site" that is either occupied or empty. We have

$$
M=2 J \text {, }
$$

where $J$ denotes an "angular momentum". One needs the quasi-spin operators 


$$
\begin{gathered}
\hat{J}_{+}=\sum_{p} C_{p,+}^{\dagger} C_{p,-}, \\
\hat{J}_{-}=\sum_{p} C_{p,-}^{\dagger} C_{p,+}, \\
\hat{J}_{z}=\sum_{p, \mu} \mu C_{p, \mu}^{\dagger} C_{p, \mu}, \\
\hat{J}^{2}=\hat{J}_{z}^{2}+\frac{1}{2}\left(\hat{J}_{+} \hat{J}_{-}+\hat{J}_{-} \hat{J}_{+}\right),
\end{gathered}
$$

where the eigenvalues of $\hat{J}^{2}$ are $J(J+1)$. The Hamiltonian of [3] is a spinflip one that reads

$$
\hat{H}=\epsilon \hat{J}_{z}-V_{s}\left(\frac{1}{2}\left(\hat{J}_{+} \hat{J}_{-}+\hat{J}_{-} \hat{J}_{+}\right)-\hat{J}\right),
$$

or, with $V=V_{s} / \epsilon \quad($ take $\epsilon=1)$,

$$
\hat{H}=\hat{J}_{z}-V\left(\frac{1}{2}\left(\hat{J}_{+} \hat{J}_{-}+\hat{J}_{-} \hat{J}_{+}\right)-\hat{J}\right) .
$$

Thus, the unperturbed ground state (ugs) $(V=0)$ becomes, in accordance with Equation (9),

$$
\left|J, J_{z}\right\rangle=|J,-M / 2\rangle
$$

of energys

$$
E_{o}=-M / 2 .
$$

Doubly inhabited $p$-sites are not permitted. $\hat{H}$ commutes with $\hat{J}^{2}$ and $\hat{J}_{z}$. This implies that the exact solution will belong to the $J$-multiplet of the ugs. The multiplet's states are designated as $|J, m\rangle$. Necessarily, one of them will minimize the energy. The pertinent $m$ value for this state depends on the interaction's coupling constant $V$. We just mentioned that for the ugs we have $m=-J=-M / 2$. Obviously, the interaction-operator $\left(\hat{J}_{+} \hat{J}_{-}+\hat{J}_{-} \hat{J}_{+}\right)$is a quasi-spin flipping one. Accordingly, this operator becomes the more "efficacious" the more similar the populations of the two-levels are.

\subsection{Phase Transitions (PT)}

As $V$ increases from zero, the ugs energy $E_{o}$ is not affected at once. It keeps its value till a critical $V$-specific value is reached, of $1 /(M-1)$. At this stage, the interacting gs instantaneously becomes $|J,-M / 2+1\rangle$. If $V$ continues growing, additional new phase transitions (PT) occur. The PT between $J_{z}=-k$ and $J_{z}=-k+1$ tales place at $V=1 /(2 k-1)$. The successive PT's end up when we reach either $J_{z}=0 \quad\left(V_{c r i t}=1\right.$ for integer $)$ ), or $J_{z}=-1 / 2 \quad\left(V_{c r i t}=1 / 2\right.$ for odd ). Accordingly, at such stage we have, independently of $J[3]$ :

$$
V_{c r i t}=1 / 2 \text { for half-integer } J \text { or } V_{c r i t}=1 \text { for integer } J \text {. }
$$

\subsection{Treatment at Finite Temperatures $T$}

Let us insist: double inhabiting a $p$-site is not allowed. As a consequence, the 
Hamiltonian matrix' size becomes $(2 J+1) \times(2 J+1)$. Remark that the only manner to get different $J$ values is to have such double occupancy [4]. Following [4], the $J=N / 2$ multiplet is the only one is to consider.

The free energy $F$ and the partition function $Z$, with $\beta$ the inverse temperature, and $k_{B}=1$ (Boltzmann) are expressed in the fashion

$$
F=-T \ln Z=-T \ln \text { Trace }(\exp (-\beta \hat{H})) \text {. }
$$

Of course, in the Trace we sum over the $J_{z}$ quantum number $m$. Since $H$ commutes with both $J$ and $J_{z}$ we have a partition function $Z$

$$
Z=\sum_{m=-J}^{m=J} \exp \left(-\beta E_{m}\right)
$$

where the energies $E_{m}$ are

$$
E_{m}=m-V\left(J(J+1)-m^{2}-J\right) .
$$

The associated probabilities $P_{m}$ read [4]

$$
P_{m}=\frac{\exp \left(-\beta E_{m}\right)}{Z},
$$

for all $m=-J,-J+1, \cdots, J-1, J$. The entropy $S$ is written as

$$
S=-\sum_{m=-J}^{m=J} P_{m} \ln P_{m}
$$

\subsection{Statistical Complexity Measure}

The authors of Ref. [7] concocted a rather adequate functional $F[P]$ of the probability distribution able to apprehend correlations in the manner of Shannon's entropy grasping of randomness. This fact constituted a breakthrough, conceptualized with the help of the definition of López-Ruiz, Mancini, and Calbet (LMC) [7] of what became called since then the statistical complexity $C$, that individualized and quantified the relative contributions of entropy and structure to the new quantifier. The structural ingredient was described by a quantity they denominated disequilibrium $D$. This statistical complexity $C$ has many applications today (for a small sample we mention Refs. [6]-[25]) $C$ is zero in the two scenarios of perfect order and maximum disorder. LMC defined $C$ as the product of Shannon's entropy $S$ and the disequilibrium $D . D$ is a measure in probability space of the distance between the PD at hand and the uniform one. One has

$$
C=S D \text {. }
$$

For our model, according to (23)), the uniform probabilities are $P^{(u)}=1 /(2 J+1)$ for all $m$ between $-J$ and $J$, so that for us here

$$
D=\sum_{m=-J}^{m=J}\left(P_{m}-P^{(u)}\right)^{2},
$$

while 


$$
S=-\sum_{m=-J}^{m=J} P_{m} \ln P_{m} .
$$

For more details regarding $C$ we recommend Refs. [7]. With regards to this model, we always deal with a number of energy levels $N=2 J+1$.

\subsection{Distance from Equilibrium Quantifiers}

We deal with the results for the present two master equations (ME) above, that are to be compared to the quasi-static (st) results that arise out of considering, always, Boltzmann-Gibbs equilibrium-situations at the temperature $T(t)$ for all $t$. As distance quantifiers we will employ two of them

- the Kullback-Leibler divergence $D_{K L}$ between two distinct probability densities (pertaining to the $\mathrm{ME}$ and the st treatments, respectively, and

- the entropy $S$.

If we generically call $Q$ to any of these two quantifiers, the distances ME-st we are talking about here are of the form

$$
\begin{gathered}
d_{Q}(t)=\frac{\left[Q_{M E}(t)-Q_{s t}(t)\right]^{2}}{Q_{M E}(t)^{2}}, \\
D_{Q}=\int_{0}^{20 \tau} \mathrm{d} t d_{Q}(t),
\end{gathered}
$$

where, obviously, the sub-index ME (or just " $M$ ") refers to master equations results, and the sub-index "st" (or just "s") to quasi-static ones.

We expect the distances from equilibrium to be sensitive to

1) Changes in the behavior of $T$ with $t$ and

2) Structural system's changes with the coupling constant $V$,

3) The speed of the heating-up and cooling-off process, regulated by the parameter $\tau$. The shorter $\tau$, the faster the speed.

\section{Main Present Results}

We will compare three treatments in his work, for $N=5$.

- the full master equation, whose results are denoted with a super-script GM or, at times, simply G,

- the simplified master equation, whose results are denoted with a super-script $\mathrm{M}$

- the quasi-static approach, the entails equilibrium with the reservoir at any time $t$ or temperature $T$, whose results are denoted with a super-script "stat", or S.

\subsection{Time Evolution}

In Figure 1 we compare the time-evolution of the occupation probability $P_{0}$ (whose values are indicated in the y-axis), of the lowest lying level " 0 " in our three theoretical treatments, that we call, in abbreviate fashion, as Stat, ME, and GME. [Our probabilities are $P_{0}, P_{1}, \cdots, P_{4}$ ] The coupling constant is $V=2$. Ar- 
tifacts of the temperature-time triangle of apex $T_{M}$ are clearly noticeable for $0<t<2 \tau$, the triangle time-basis. For greater times rapid equilibration takes place, but in noticeably quicker fashion for GM than for M. Note the peak of $P_{0}$ in the ME treatment at $t=2 \tau$, where the temperature ceases changing.

Figure 2 is identical to Figure 1 save for the fact that here $V=0$ (interaction-less system). The appearances are different, but still rapid equilibration takes place, but in quicker fashion for GM than for M. Of course, in an interactionless system, added heat makes $P_{0}$ to diminish, while the fermion-fermion interaction in Figure 1 makes it to grow. The couple of Figure 1, Figure 2 beautifully illustrate the competition heat-interaction.

\subsection{Distance Quantifiers}

\subsubsection{Kullback-Leibler Divergence $D_{K L}$}

We consider now the $D_{K L}$ divergence. We consider both the interaction and interaction-less situations, in Figure 3 and Figure 4, respectively. We contemplate three divergence types between

1) $M$ and $S$

2) $G$ and $S$

3) $G$ and $M$ and vice versa.

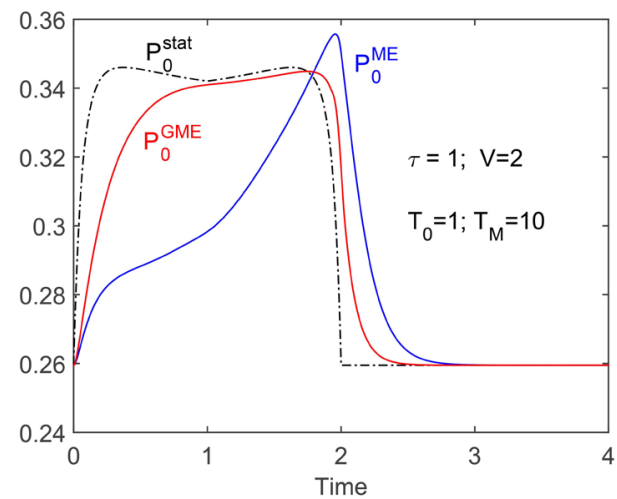

Figure 1. $V=2$. Time evolution of $P_{0}$ in our 3 treatments.

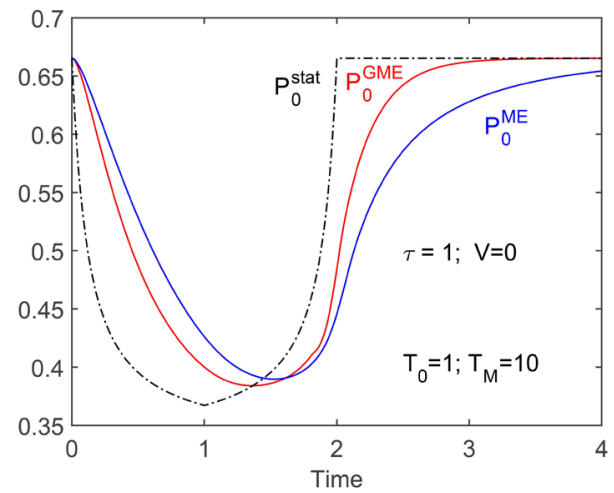

Figure 2. $V=0$. Time evolution of $P_{0}$ in our 3 treatments. 

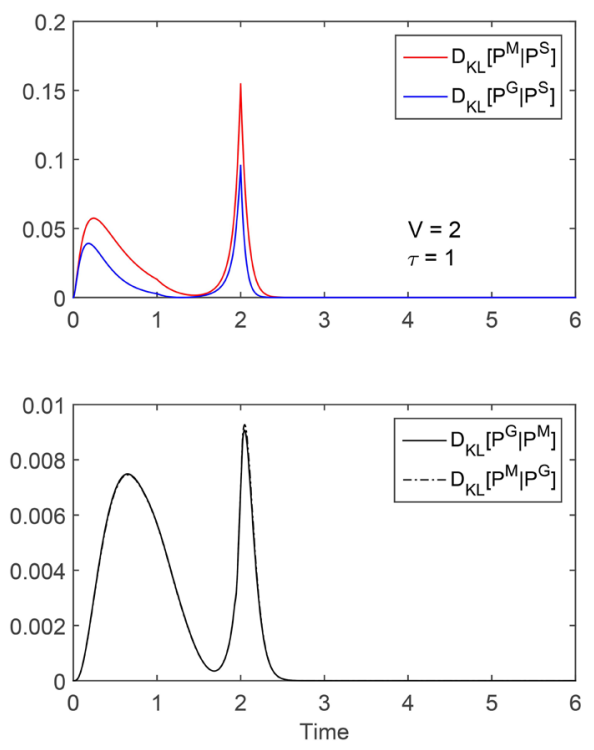

Figure 3. $V=2 . D_{K L}$ distances.
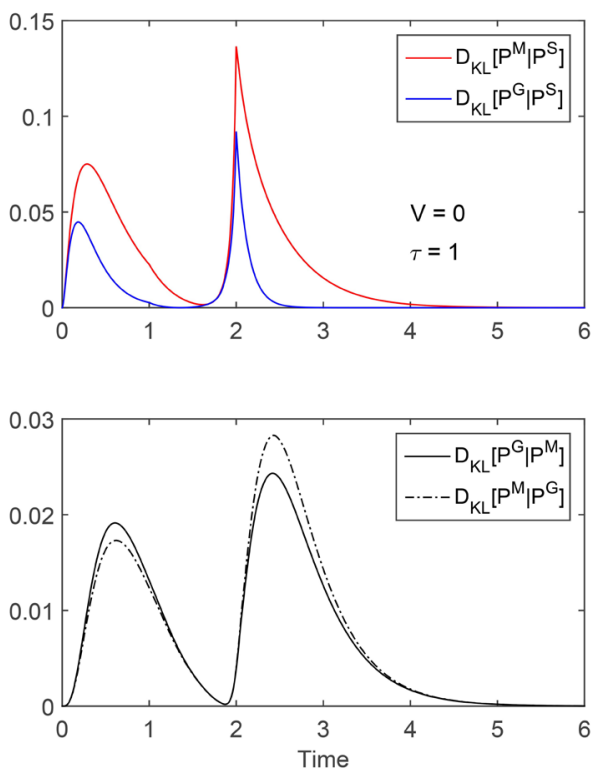

Figure 4. $V=0 . D_{K L}$ distances.

Consider the case $V=2$ (Figure 3 ). We see again that $\mathrm{G}$ reaches equilibrium quicker than $M$. One can not distinguish, though, between the GM or the MG divergences, a fact that must be discussed in conjunction with its Figure 4 counterpart. There, the same happens for $V=0$ in (1) and (2), but the difference are larger here. The G-M and M-G divergences noticeably differ now, while they were indistinguishable for the interacting system (Figure 3). We see that the competition heat-fermion-fermion interaction makes the different types of description more distinguishable when $V=0$. To repeat, we conclude that the differences between the full master equation and the reduced one are smaller when the fermions interact among themselves than when they are free. 


\subsubsection{Entropies and Entropic Distances (28)}

Our quantifiers are entropic now. We will again encounter that the differences between the full master equation and the reduced one are smaller when the fermions interact among themselves than when they are free.

We consider here, for $N=5$, the interaction $V=2$ and interaction-less $V=0$ scenarios in, respectively, Figure 5 and Figure 6 . Entropies behave in rather similar fashion in our three treatments. Not so the entropic distances. In particular for free fermions the differences persists long after the thermal disturbance has ceased to exist. The full master equation leads to more rapid equilibration than the incomplete one.
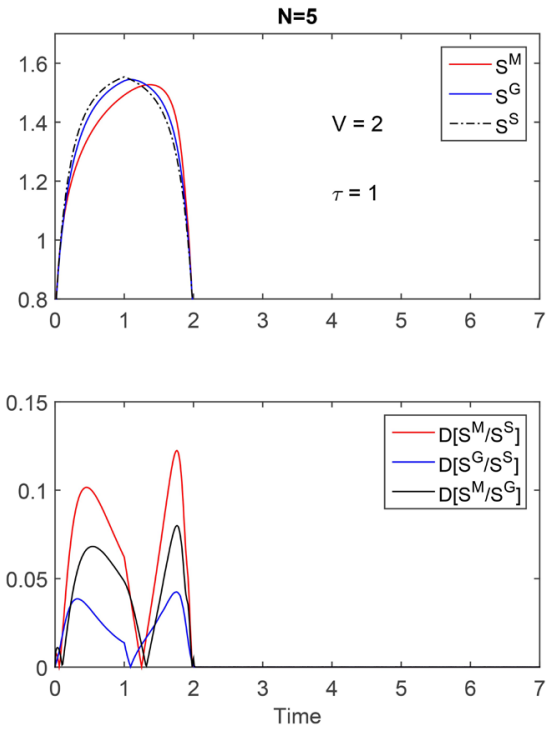

Figure 5. $V=2$. Three entropies and entropic distances.
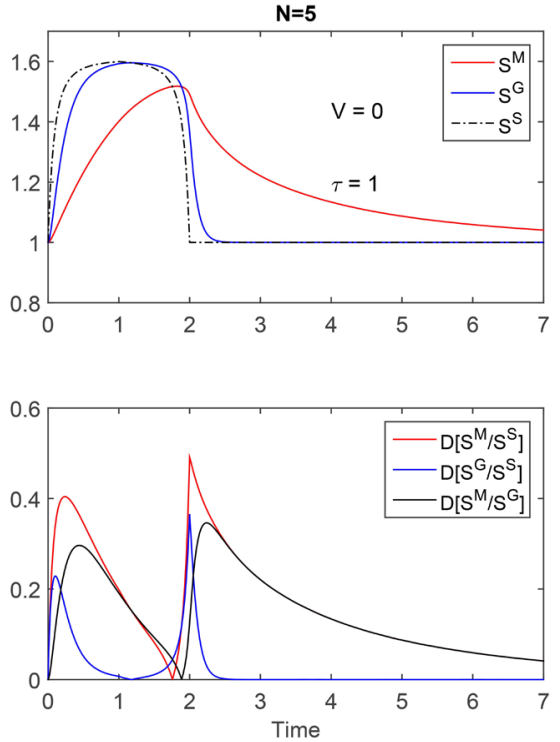

Figure 6. $V=0$. Three entropies and entropic distances. 


\subsection{3. $\tau$-Dependence}

We study here some facets of our results' dependence on the duration $2 \tau$ of our triangular temperature disturbance for distinct $N$-values. Take a glance now at Figure 7. We use there three different $\tau$-values and look at the entropic distances M-S and G-S for $V=1$. While the M-S distances behave rather erratically, the G-S ones tend to rapidly converge, in faster fashion the larger the $\tau$-value (here, our largest value equals five). The reason for this behavior is clear. The largest $\tau$ corresponds to the smoother temperature change. Note also that, the larger $N$ becomes, the more noticeable the manner in which the entropic distances diminish.

\subsection{4. $N$-Dependence}

We look in Figure 8 at the $N$-dependence of the two master equations G (complete) and $\mathrm{M}$ (operating selection rules that forbid most possible transitions). More precisely, at the dependence on the number of fermions $\mathcal{N}=2 N+1$ The $\mathrm{G}$ and $\mathrm{M}$ treatments behave in quite different fashion, with regards to both

1) difference between the interaction and interaction-less scenarios,

2) difference between the $M$ and $G$ master equations deportment.

We clearly notice that

- for interacting fermions the entropic distance to the equilibrium instance rapidly decreases as $N$ grows,

- The $\mathrm{G}$ master equations leads to equilibrium much more rapidly than the incomplete $\mathrm{M}$ one.

- Equilibration proceeds more rapidly for the interacction that for the interaction-less system.
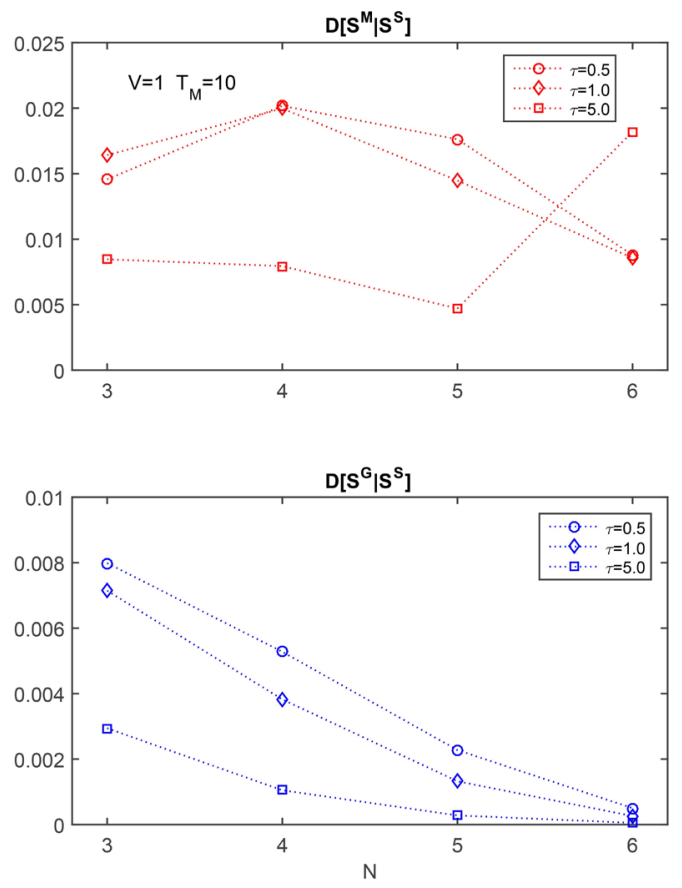

Figure 7. Behavior of the entropic distances for three different $\tau$ values. 

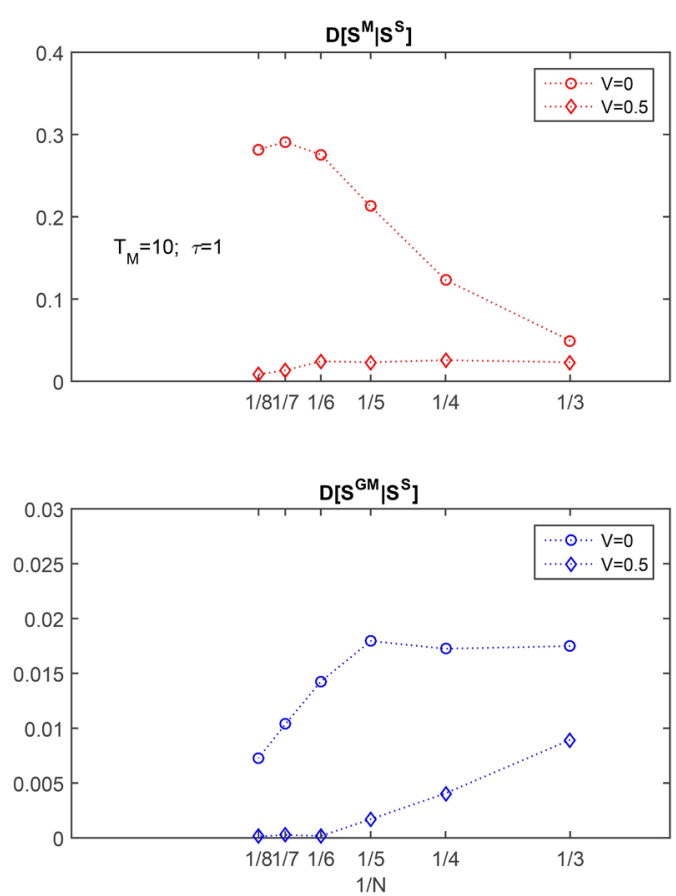

Figure 8. Behavior of the entropic distances for different numbers of fermions $\mathrm{N}$.

\section{Conclusions}

Our central issue in this effort was the investigation of just how a master equation leads to an equilibrium scenario, with reference to a set of mutually interacting fermions. They are subjected here to a spin-flip interaction. Two fermions enter the Feynman diagram, interact (coupling constant $V$ ), and come out with their spins reversed. This problem can be tackled in analytic fashion.

The fermionic system is in thermal contact with a heath-bath at a moveable temperature $T$. The reservoir first heats up from an initial temperature $T_{=}$, reaches a maximum temperature $T_{M}$, and then cools-off, returning to its initial state of temperature $T_{0}$. An appropriate master equation describes the process. Some time after the $T$-disturbance has ceased the system reaches equilibrium with the reservoir.

Three ways of tackling the description were used.

1) A quasi-static approach in which we contemplate at all times a canonical equilibrium Gibbs-density distribution at the extant temperature $T$, whatever it may be.

2) A complete master equation (ME) in which all possible fermion-energy levels' populations interact amonst themselves.

3) A reduced ME in which suitable selection rules only permit a population level to interact only with a few others.

We compare the three description manners both for the interacting system and for the free one of $V=0$. We concoct some distances' measure between the three different ensuing time-dependent probability densities. Our main observa- 
tions are:

- The $T$ change's effects are significantly diminished by the fermion-fermion interaction.

- The time-evolution of the distance between the two pertinent probability distributions is rather sensitive to the details of the heating-cooling process.

- The larger the number of interacting fermions, the sooner the system reaches equilibrium. This is for us a rather surprising finding that we regard as our main one here.

- The fermion-fermion interaction always favors a more rapid equilibration.

\section{Conflicts of Interest}

The authors declare no conflicts of interest regarding the publication of this paper.

\section{References}

[1] Kryszewski, S. and Czechowska-Kryszk, J. (2008) Master Equation-Tutorial Approach.

[2] Takada, A., Conradt, R. and Richet, P. (2013) Journal of Non-Crystalline Solids, 360, 13. https://doi.org/10.1016/j.jnoncrysol.2012.10.002

[3] Plastino, A. and Moszkowski, S.A. (1978) Il Nuovo Cimento, 47, 470. https://doi.org/10.1007/BF02896236

[4] Pennini, F. and Plastino, A. (2018) Physica A, 491, 305. https://doi.org/10.1016/j.physa.2017.09.041

[5] Ziman, J. (2002) Real Science. Cambridge Univ. Press, Cambridge.

[6] Plastino, A.R., Ferri, G.L. and Rocca, M.C. (2020) Journal of Modern Physics, 11, 1. https://doi.org/10.4236/jmp.2020.111001

[7] Lpez-Ruiz, F.R., Mancini, H. and Calbet, X. (1995) Physics Letters A, 209, 321. https://doi.org/10.1016/0375-9601(95)00867-5

[8] Martin, M.T., Plastino, A. and Rosso, O.A. (2003) Physics Letters A, 311, 126. https://doi.org/10.1016/S0375-9601(03)00491-2

[9] Kowalski, A., Martin, M.T., Plastino, A., Proto, A. and Rosso, O.A. (2003) Physics Letters A, 311, 180. https://doi.org/10.1016/S0375-9601(03)00470-5

[10] Ribeiro, H.V., Zunino, L., Lenzi, E.K. Santoro, P.A. and Mendes, R.S. (2012) PLoS ONE, 7, e40689. https://doi.org/10.1371/journal.pone.0040689

[11] Ribeiro, H.V., Zunino, L., Mendes, R.S. and Lenzi, E.K. (2012) Physica A, 391, 2421. https://doi.org/10.1016/j.physa.2011.12.009

[12] Manzano, D. (2012) Physica A, 391, 6238. https://doi.org/10.1016/j.physa.2012.06.058

[13] Borgoo, A., Geerlings, P. and Sen, K.D. (2011) Physics Letters A, 375, 3829. https://doi.org/10.1016/j.physleta.2011.09.031

[14] Dehesa, J.S., Lpez-Rosa, S. and Manzano, D. (2009) The European Physical Journal $D$, 55, 539. https://doi.org/10.1140/epjd/e2009-00251-1

[15] Esquivel, R.O., Molina-Espritu, M., Angulo, J.C., Antoln, J., Flores-Gallegos, N. and Dehesa, J.S. (2011) Molecular Physics, 109, 2353.

https://doi.org/10.1080/00268976.2011.607780 
[16] Lpez-Ruiz, R. (2013) Concepts and Recent Advances in Generalized Information Measures and Statistics. Bentham Science Publishers, Sharjah, 147-168.

[17] Sen, K.D. (2020) Quantum Representative, 20, 313.

[18] Martin, M.T., Plastino, A. and Rosso, O.A. (2006) Physica A, 368, 439. https://doi.org/10.1016/j.physa.2005.11.053

[19] Pennini, F. and Plastino, A. (2017) Physics Letters A, 381, 212. https://doi.org/10.1016/j.physleta.2016.11.023

[20] Pennini, F. and Plastino, A. (2018) Physica A, 506, 828. https://doi.org/10.1016/j.physa.2018.05.003

[21] Pennini, F. and Plastino, A. (2017) Physica A, 488, 85. https://doi.org/10.1016/j.physa.2017.07.005

[22] Pennini, F. and Plastino, A. (2019) Entropy, 21, 558. https://doi.org/10.3390/e21060558

[23] Branada, R., Pennini, F. and Plastino, A. (2018) Physica A, 511, 18. https://doi.org/10.1016/j.physa.2018.07.037

[24] Pennini, F. and Plastino, A. (2018) Physica, 491, 305. https://doi.org/10.1016/j.physa.2017.09.041

[25] Pennini, F. and Plastino, A. (2017) Physics Letters A, 381, 3849-3854. https://doi.org/10.1016/j.physleta.2017.10.025 